\title{
1 Swift Large-scale Examination of Directed Genome Editing (SLEDGE Hammer)
}

2 Omar T. Hammouda ${ }^{1,2}$, Thomas Thumberger ${ }^{1}$, Joachim Wittbrodt ${ }^{1}$

$3{ }^{1}$ Centre for Organismal Studies Heidelberg, Heidelberg University, Im Neuenheimer

4 Feld 230, 69120 Heidelberg, Germany.

$5 \quad{ }^{2}$ Heidelberg Biosciences International Graduate School, Heidelberg University, Im 6 Neuenheimer Feld 501, 69120 Heidelberg, Germany.

\section{Abstract}

9 In the era of CRISPR gene editing and genetic screening, there is an increasing demand for quick and reliable nucleic acid extraction pipelines for rapid genotyping of

11 large and diverse sample sets. Despite continuous improvements of current workflows, the handling-time and material costs per sample remain the major limiting factors. Here we present a robust method for low-cost DIY-pipet tips addressing these needs; i.e. using a cellulose filter disc inserted into a regular pipet tip. These 15 filter-in-tips allow for a rapid, stand-alone three-step genotyping workflow by simply binding the DNA contained in the primary lysate to the cellulose filter, washing it in water and eluting it directly into the buffer for the downstream application (e.g. PCR). This drastically cuts down processing time to maximum 30 seconds per sample, with the potential for parallelizing and automation. We show the ease and sensitivity of our procedure by genotyping genetically modified medaka and zebrafish embryos (targeted CRISPR/Cas9 knock-out and knock-in) in a 96-well plate format. The robust isolation and detection of multiple alleles of various abundancies in a mosaic genetic background allows phenotype-genotype correlation already in the injected generation, demonstrating the reliability and sensitivity of the filter-in-tips.

25 Furthermore, our method is applicable across kingdoms with samples ranging from 
cells to tissues (e.g. plant seedlings, adult flies, mouse cell culture and tissue as well as adult fish fin-clips).

\section{Introduction}

Gene-editing tools such as CRISPR/Cas9 have revolutionized genome editing in most model organisms (Cong et al., 2013; Mali et al., 2013; Stemmer et al., 2015). The easy application of this genome targeting method in organisms as well as cell culture allows extensive high-throughput applications such as genetic screens (Baranski et al., 2018; Kweon and Kim, 2018). Common to all genome targeting applications is the need for subsequent genotyping. Following PCR amplification of the targeted loci, among the most commonly used assays for indel detection are mismatch cleavage assays (using T7 Endonuclease I or Cel-based Surveyor assay), high-resolution melting analysis (HRMA) and sequencing (Thomsen et al., 2012; Zischewski et al., 2017). However, the high-throughput approaches face a bottle neck when it comes to genomic DNA extraction which usually requires a time and material consuming protocol.

Genome extraction has greatly improved due to the numerous advances on nucleic acid purification methods. These either rely on solid-phase extraction kits or on traditional phenol-chloroform purification, both of which are time or material consuming and require a lot of steps (Ali et al., 2017). Recent publications have shown the ability of membranes of different materials to bind nucleic acids with high affinity, thus eliminating the need for nucleic acid purification by direct amplification of the nucleic acid off the membrane (Jangam et al., 2009; Kim et al., 2010; McFall et al., 2015). A recent improvement has greatly reduced the genome extraction step to 
52 under 30 seconds using cellulose paper-based dipsticks (Zou et al., 2017). Although

53 extremely advantageous in terms of time and material, these methods require

54 fabrication or manufacturing steps, which make them unsuitable for high-throughput applications, especially for automated systems.

Teleost fish such as the Japanese rice fish medaka (Oryzias latipes) have been

58 vertebrate model organisms for more than a century (Wittbrodt et al., 2002).

59 Numerous advantages such as high fecundity and fast generation times;

60 transparency of chorion and body allow for live non-invasive in vivo imaging; and the availability of various genetic tools makes them an attractive model organism for developmental, genetic and molecular studies (Kirchmaier et al., 2015). Moreover, the high efficiency of genome-editing in medaka by CRISPR/Cas9 renders it very

64 attractive for high-throughput in vivo screening applications (Stemmer et al., 2015).

65 However, despite the continuous development and progression of high-throughput 66 automated imaging and analysis pipelines, current genotyping protocols display the 67 limiting step.

In this study, we have developed a very fast, simple and efficient genotyping 70 protocol, which has great potential for high-throughput and automated applications whenever it comes to nucleic acid extraction and transfer. In a medaka and zebrafish context, we describe our Swift Large-scale Examination of Directed Genome Editing 73 (SLEDGE Hammer) protocol. Here we demonstrate rapid genotyping in a 96-well based format of various CRISPR/Cas9 edited medaka and zebrafish embryos. In brief, a cellulose paper disc inserted into conventional pipet tips allows for skipping 76 purification of genomic DNA from cell/tissue lysates and facilitates direct use with 77 PCR buffers. Our filter-in-tip approach allows easy high-throughput upscaling by 
78 using multichannel pipets to handle several samples simultaneously; while also

79 offering potential for automated pipetting systems suitable for high-throughput automated screens. The filter-in-tips are generally applicable when transfer of nucleic acids is aimed for as shown by genomic DNA extraction from varying sources of tissues derived from diverse (model) organisms such as Arabidopsis thaliana, Chironomus riparius, Drosophila melanogaster, Mouse ear punches, Mouse

\section{Embryonic stem cells, fish embryos and fin-clips.}

\section{Results and Discussion}

87
Teleost fish have been extensively used for high-throughput approaches (Kithcart and MacRae, 2017; Lessman, 2011; Oxendine et al., 2006; Spomer et al., 2012) due to their small sized embryos, transparency and biological relevance as vertebrates. Recently, there have been various key developments in medaka research: 1) the improved efficiency of medaka genome editing via CRISPR/Cas9 (Stemmer et al., 2015); and 2) the establishment of the first wild vertebrate medaka inbred panel (Spivakov et al., 2014), which is the basis for phenotype / population genomic studies that rely on automated phenotyping as well as reliable and precise genotyping.

These developments allow us to foresee a surge in the use of medaka for various high-throughput assays (e.g. genetic screens, drug screens and Genome Wide Association Studies). However, genotyping a large number of specimens still remains an obstacle in terms of time and effort, as standard genotyping protocols start with grinding of embryos/tissue in lysis buffer and incubating for an extended period of time, followed by genomic DNA extraction and precipitation (most commonly using Isopropanol). Precipitation however comes with the risk of losing the genomic DNA 
104 sample. Taken together, this purification procedure can easily take 2 days and is

105 hence not feasible in a high-throughput screening context.

106

107 In the light of the reported nucleic acid binding property of cellulose based filter paper

108 (Zou et al., 2017), we sought to exploit this feature in order to by-pass the tedious

109 genomic DNA purification step, thus saving time and material. To this end, we used a

110 paper puncher to create Whatman filter paper discs $(\approx 2 \mathrm{~mm})$ and placed them inside

111 of standard yellow $200 \mu$ tips (filter-in-tips; Fig. 1A). To test the applicability of these

112 modified pipet tips for rapid genotyping, the fins of 3 male and 3 female adults were

113 clipped and administered to rapid genomic DNA extraction for sex determination by

114 PCR. Fin clips were put into $100 \mu$ l fin-clip buffer-containing Eppendorf tubes. $50 \mu \mathrm{l}$ of

115 PCR mix with primers for the non-muscle actin b (actb) serving as control and dnmtY

116 for genomic male sex determination were prepared for each specimen. After short

117 grinding of the tissue, the filter-in-tips were used to soak the Whatman paper disc

118 with the fin-clip lysate for the nucleic acids to bind to the cellulose simply by pipetting

119 the liquid up and letting it rest in the tip for $\approx 10$ seconds. Subsequently the lysate was

120 released back into the tube for storage. Next, the filter was washed to remove lysate

121 buffer reagents (which impair later diagnostics) by brief 1-2 washing steps in MilliQ

122 water (individual aliquots per tip) by pipetting up and down. Finally soaking the filter

123 disc for $\approx 5-10$ seconds in the PCR reaction mix by pipetting up with the very same

124 filter-in-tip released enough material for amplification. Whereas the $d n m t Y$ band

125 correlated with male sex phenotype, actb control amplicon was present in all 126 specimens (Fig. 1B).

128 We next raised the question if this SLEDGE-Hammer protocol is robust and reliable 129 for large scale individual extractions of genomic DNA by equipping a multichannel 
130 pipettor with the filter-in-tips (Fig. 1 C). And further, if our protocol would allow to

131 detect minor nucleotide changes as to be found in mosaic embryos resulting from

132 CRISPR/Cas9-mediated knock-out. We thus targeted the oculocutaneous albinism 2

133 (oca2, Fig. 1D) gene responsible for melanin pigmentation using three individual

134 sgRNAs in combination with Cas9 mRNA and gfp mRNA as injection marker in

135 medaka and zebrafish one cell stages. Loss of pigmentation requires a bi-allelic

136 mutation to occur in the oca2 open reading frame, rendering this gene a perfect

137 candidate for CRISPR/Cas9 knock-out efficiency analysis (Lischik et al., 2018). In

138 addition to the knock-out attempts caused as a result of the error-prone non-

139 homologous end-joining (NHEJ) repair mechanism, a more delicate CRISPR/Cas9

140 approach for tagging the Retinal homeobox protein $2(R x 2)$ gene with gfp was used

141 (Fig. 1D). A biotinylated dsDNA PCR donor fragment comprising the gfp sequence

142 flanked by nucleotide stretches homologous to about 400bp up and downstream of

143 the $r \times 2$ sgRNA target site was co-injected with Cas9 mRNA and the respective rx2

144 sgRNA (Gutierrez-Triana et al., 2018). Per oca2 sgRNA, 21 positively injected

145 embryos (4 days post fertilization (dpf) for medaka, $2 \mathrm{dpf}$ for zebrafish) were

146 randomly selected and put into a $96 \mathrm{U}$-well plate for phenotype/genotype screening.

147 For screening of gfp-rx2 integration, 21 injected embryos were randomly picked at 2

$148 \mathrm{dpf}$ and administered alike (Supplementary Fig. S1A). One negative control (empty

149 well containing embryo rearing medium) as well as two uninjected controls

150 complemented each group.

151

152 Phenotyping revealed that the knock-out attempts in the oca2 genes caused the 153 expected loss of pigmentation in all specimens with various extents as evident in the 154 retinal pigmented epithelium (RPE; Fig. 1E). Tagging of the rx2 gene was visible by 155 specific GFP expression in the medaka retinae (Fig. 1E). HDR-mediated knock-in 
156 attempts are less efficient than knock-out approaches, still $\approx 40 \%(8 / 21)$ of injected

157 specimens showed varying numbers of GFP positive cells in the developing eye. To

158 correlate with the observed phenotype, each embryo was genotyped individually.

160 Based on the 96 well plate format used for long term single-embryo monitoring / 161 phenotyping, we additionally have created a stainless-steel 96-pin mortar (a.k.a. 'the 162 Hammer'). After phenotyping (plate imaging), the fish medium in the $96 \mathrm{U}$-well plate 163 was replaced with fin-clip lysis buffer (100 $\mu$ l per well) and the embryos were 164 simultaneously ground using our custom-made mortar. For individual genotyping we 165 followed the rapid protocol described above using the filter-in-tips in combination with a multichannel pipet (Fig. 1C). For the oca2 knock-out embryos, locus amplification 167 revealed that in all cases transfer of genomic DNA to the PCR mixture was 168 successful (Fig. 1F, Supplementary Fig. S1B). We subsequently examined the 169 success of targeted genome editing (indel formation) in the oca2 loci using T7 170 Endonuclease I (T7EI) assay, which detects and cuts heteroduplexes caused by a 171 heterogeneous mixture of indel alleles from genome targeted specimens. T7EI 172 genotyping confirmed the phenotypic observation, i.e. all injected embryos of all oca2 173 knock-out attempts showed heteroduplex digestion in the T7EI assay, whereas the 174 locus amplicon of the respective uninjected controls did not (Fig. 1F, Supplementary 175 Fig. S1C). Furthermore, all gfp-rx2 tagging approaches that were positive in the GFP 176 screening could as well be confirmed by PCR genotyping as fusion of the gfp 177 sequence with the rx2 locus in the respective specimens (Fig. 1F, Supplementary 178 Fig. S1D-E). It is interesting to note that in 7 out of the 8 positively screened 179 embryos, a PCR band indicative for single-copy HDR-mediated integration was 180 evident (Fig. 1F, Supplementary Fig. S1D) whereas the integration of the donor 
181 cassette probably underwent NHEJ-mediated concatenation and integration in four

182 cases (Supplementary Fig. S1E).

183

184 Besides genotype/phenotype confirmation, all PCRs and T7El assay revealed that 185 the filter-in-tip approach robustly and reliably extracted genomic DNA for PCR 186 amplification and that the transferred genomic DNA contained enough allele variants

187 for the subsequent T7EI assay. Furthermore, considering genotyping of 96 embryos, 188 conventional genomic DNA extraction could easily have taken 2 days and would 189 require tedious and repetitive pipetting. In contrast, our SLEDGE-Hammer protocol 190 allows drastic shortening of time and pipetting steps to a maximum of 10-15 minutes 191 per plate using a standard 8-channel multipipettor. This effortless genotyping will 192 massively upscale with the continuous advancements of automated multipipettors, 193 which have now reached up to 384 channels.

195 Besides the reliable extraction of genomic DNA from fish embryos, we investigated if 196 this method can be generalized for genomic DNA extraction from varying sources of 197 tissues derived from (model) organisms such as Arabidopsis thaliana, Drosophila 198 melanogaster, Chironomous riparius, mouse ear punches (EP) and mouse 199 embryonic stem cells (mESC) (Fig. 2). In all cases, grinding of tissue, i.e. a single 200 seedling of Arabidopsis, an individual adult fly of both insect species as well as an 201 individual mouse ear punch and $2.5 \times 10^{5}$ ESC suspension in lysis buffer and 202 subsequent transfer of genomic DNA by pipetting of the lysate using our filter-in-tips transferred enough material for specific locus amplification.

205 Taken together, our SLEDGE Hammer protocol with the adapted filter-in pipet tips, 206 allows to bypass the otherwise tedious and time-consuming genomic purification step 
207 that hitherto limited high-throughput genotyping approaches. With the rapid

208 genotyping method presented here, future attempts of individual phenotype-genotype

209 correlation following high-throughput automated screening of individual specimens is

210 in reach. Furthermore, our simple modification of already available pipet tips can

211 easily be adapted for use in fully automated research applications involving almost

212 any biological sample. Due to the drastic reduction in time and costs, we further

213 foresee this application for stock maintenance in laboratories or stock centers, where

214 genotyping is a daily routine.

215

216 Materials and Methods

217

218 Ethics Statement

219 All fish are maintained in closed stocks at Heidelberg University. Medaka (Oryzias

220 latipes) and zebrafish (Danio rerio) husbandry (permit number 35-9185.64/BH

221 Wittbrodt) were performed according to local animal welfare standards

222 (Tierschutzgesetz $\S 11$, Abs. 1, Nr. 1) in accordance with European Union animal

223 welfare guidelines (Bert et al., 2016). The fish facility is under the supervision of the

224 local representative of the animal welfare agency. Embryos of medaka of the

225 wildtype $\mathrm{Cab}$ strain and of zebrafish $\mathrm{AB} / \mathrm{AB}$ line were used at stages prior to 226 hatching. Medaka were raised and maintained as described previously (Köster et al., 227 1997).

228

229

sgRNA target site selection and in vitro transcription

230 sgRNAs were designed with CCTop as described in Stemmer et al. (Stemmer et al., 231 2015). sgRNA for $r x 2$ was the same as in Stemmer et al. (Stemmer et al., 2015) and 232 the olOca2 sgRNAs were the same as in Lischik et al. (Lischik et al., 2018). The 
233 following target sites were used (PAM in brackets): rx2 T1

234 (GCATTTGTCAATGGATACCC[TGG]), olOca2

235 (GAAACCCAGGTGGCCATTGC[AGG]), $\quad$ olOca2 T2

236 (TTGCAGGAATCATTCTGTGT[GGG]), drOca2 T1

237 (GTACAGCGACTGGTTAGTCA[TGG]). Cloning of sgRNA templates and in vitro

238 transcription was performed as detailed in Stemmer, et al. (Stemmer et al., 2015).

240 Microinjection

241 Medaka one-cell stage embryos were injected in the cytoplasm as previously

242 described (Stemmer et al., 2015), zebrafish one-cell stage embryos were injected in

243 the cytoplasm or yolk. Injection solutions for oca2 targeting comprised: $150 \mathrm{ng} / \mu \mathrm{l}$

244 Cas9 mRNA, $15 \mathrm{ng} / \mu$ respective sgRNA (either olOca2 T1, olOca2 T2 or drOca2 T1)

245 and $10 \mathrm{ng} / \mu \mathrm{l}$ gfp mRNA as injection tracer. Injected embryos were incubated at $28^{\circ} \mathrm{C}$

246 and screened for GFP expression at $1 \mathrm{dpf}$. Injection solution for gfp-rx2 tagging

247 comprised $150 \mathrm{ng} / \mu \mathrm{l}$ Cas $9 \mathrm{mRNA}, 15 \mathrm{ng} / \mu \mathrm{l} r \times 2$ T1 sgRNA and $5 \mathrm{ng} / \mu \mathrm{l}$ biotinylated

248 PCR donor fragment (Gutierrez-Triana et al., 2018).

250 Sample preparation and Imaging

251 Medaka and zebrafish embryos were administered to a 96 U-well microtiter plate

252 (Nunc, Thermofisher \#268152) containing either 1x embryo rearing medium or $1 \mathrm{x}$ 253 zebrafish medium for automated screening/phenotyping using an ACQUIFER 254 Imaging Machine (DITABIS AG, Pforzheim, Germany). Images were acquired in 255 brightfield using 9 z-slices $(d z=100 \mu \mathrm{m}$ ) and a 4x Plan UW N.A. 0.06 (Nikon, 256 Düsseldorf, Germany) to capture the centered embryo. Integration times were fixed 257 with $100 \%$ relative white LED intensity and 50 ms exposure time. GFP channel was 258 used on rx2 specimens at $30 \%$ relative LED intensity and 200 ms exposure time. 


\section{Nucleic acid extraction and locus amplification by PCR}

261 Fin-Clip Lysis Buffer was used for medaka, zebrafish, A. thaliana, C. riparius and $D$.

262

263

264

265

266

267

268

269

270

271

272

273

274

275

276

277

278

279

280

281

282

283

melanogaster (0.4 M Tris-HCl pH 8.0, 5 mM EDTA pH 8.0, 0.15 M NaCl, 0.1\% SDS

in Milli-Q water). For $M$. musculus ear clip and embryonic stem cell samples $1 \%$

SDS Lysis Buffer was used (0.1 M Tris- $\mathrm{HCl}$ pH 8.0, 5 mM EDTA pH 8.0, 0.2 M NaCl, $1 \%$ SDS in Milli-Q water).

For medaka and zebrafish extraction of genomic DNA, see main text. Our 96-well Uplate adapted stainless-steel 96-pin mortar was pre-cleaned by incubation in hypochlorite solution (1:10 dilution of commercial bleach reagent) for at least 15 minutes followed by 5 minutes incubation in MilliQ water. Plate with embryo lysate can be optionally stored at $4^{\circ} \mathrm{C}$ with proper plate seal. $2 \mathrm{~mm}$ diameter paper puncher (Harris Uni-Core 2.0) was used on Whatman Cellulose paper (3030-917 Grade 3MM) to produce the paper discs which were transferred directly from the puncher to standard yellow $200 \mu$ pipet tips (Steinbrenner $\mathrm{GmbH}$ ). $50 \mu \mathrm{l}$ PCR reaction mixes for the amplification of the desired genomic loci were prepared on ice using 1x Q5 reaction buffer, $200 \mu \mathrm{M}$ dNTPs, $200 \mu \mathrm{M}$ primer forward and reverse and $0.6 \mathrm{U} / \mu \mathrm{l}$ Q5 polymerase (New England Biolabs). 30 PCR cycles were run in all samples except for $A$. thaliana, $C$. riparius and D. melanogaster (35 cycles); annealing temperatures, extension times and primer sequences are given and PCR conditions used are listed in Table 1. Our filter-in-tips were used to transfer nucleic acids from tissue lysate to the PCR mix in three simple steps. 1) Binding: pipetting enough tissue lysate (here $50 \mu \mathrm{l})$ to soak the paper disc and wait $\approx 10$ seconds before releasing the lysate back. 2) Washing: 1-2 washing steps by brief up and down pipetting in MilliQ water, the higher the concentration of SDS the more washing steps are required, i.e. for $1 \%$ or 
285 higher SDS containing buffers, 4 washing steps are advised. 3) Elution: pipetting up

286 the PCR mix to soak the paper disc (here $50 \mu \mathrm{l}$ ), wait $\approx 5-10$ seconds and release the

287 PCR mix which will contain enough nucleic acids to be amplified. Following PCR, 10

$288 \mu$ l PCR reaction $+2 \mu$ l 6x orange Loading Dye were loaded on $1.5 \%$ Agarose in

289 1xTAE gels. Gel electrophoresis was performed at $90 \mathrm{~V}$.

290

291 Table 1. List of primers and PCR conditions used

292 All primers used in this study are given in 5'-3' direction. Expected band size

293 displayed. Note: for gfp-rx2 two major bands are expected upon HDR-mediated

294 integration - the wt size of non-gfp-integrated loci as well as the larger single-gfp

295 insertion. $R \times 2$ tagging design is as described in Gutierrez et al., 2018.

\begin{tabular}{|c|c|c|c|c|c|}
\hline organism & gene & primers ( $5^{\prime}-3^{\prime}$ direction) & $\begin{array}{c}\text { annealing } \\
\text { temp. }\end{array}$ & $\begin{array}{l}\text { extension } \\
\text { time }\end{array}$ & $\begin{array}{l}\text { band } \\
\text { size }\end{array}$ \\
\hline \multirow{2}{*}{$\begin{array}{c}\text { Arabidopsis } \\
\text { thaliana }\end{array}$} & tubulin Lf & AGTAGTTTAAGGACCTACTTCG & \multirow{2}{*}{$60^{\circ} \mathrm{C}$} & \multirow{2}{*}{14 secs } & \multirow{2}{*}{$467 \mathrm{bp}$} \\
\hline & tubulin Lr & GAGCCTTACAACGCTACTCTGTCTGTC & & & \\
\hline \multirow{2}{*}{$\begin{array}{c}\text { Chironomous } \\
\text { riparius }\end{array}$} & germ cell-less Lf & СTTTATTAGCGTTCGGTCGTG & \multirow{2}{*}{$63^{\circ} \mathrm{C}$} & \multirow{2}{*}{35 secs } & \multirow{2}{*}{$1000 \mathrm{bp}$} \\
\hline & germ cell-less Lr & CСAACGTAАСАTTATCСTTCGC & & & \\
\hline \multirow{2}{*}{$\begin{array}{c}\text { Drosophila } \\
\text { melanogaster }\end{array}$} & twist Lf & CAATTTGAGCAATGGCCGGAAGGA & \multirow{2}{*}{$70^{\circ} \mathrm{C}$} & \multirow{2}{*}{28 secs } & \multirow{2}{*}{$931 \mathrm{bp}$} \\
\hline & twist Lr & ACTGCTGCTGCTGGTTGTTGTAGA & & & \\
\hline \multirow{3}{*}{$\begin{array}{c}\text { Mus } \\
\text { musculus }\end{array}$} & trpm4 P1 & GTTTGATGTCTCCTTCAGTCG & \multirow{3}{*}{$62{ }^{\circ} \mathrm{C}$} & \multirow{3}{*}{6 secs } & \multirow{3}{*}{200 bp } \\
\hline & trpm4 P2 & GAGTTCCTGTCCTCCTAAAGG & & & \\
\hline & trpm4 P3 & ACCTACAGGAAACCTCGGGG & & & \\
\hline \multirow{2}{*}{ Danio rerio } & drOca2 Lf & ACAGGTGCTGTATAATTGGACCAT & \multirow{2}{*}{$65^{\circ} \mathrm{C}$} & \multirow{2}{*}{19 secs } & \multirow{2}{*}{$619 \mathrm{bp}$} \\
\hline & drOca2 Lr & AAAGAGTGGTCATAAACGGCTACT & & & \\
\hline \multirow{10}{*}{$\begin{array}{l}\text { Oryzias } \\
\text { latipes }\end{array}$} & olOca2 Lf & GTTAAAACAGTTTCTTAAAAAGAACAGGA & \multirow{2}{*}{$62{ }^{\circ} \mathrm{C}$} & \multirow{2}{*}{21 secs } & \multirow{2}{*}{$696 \mathrm{bp}$} \\
\hline & olOca2 Lr & AGCAGAAGAAATGACTCAACATTTTG & & & \\
\hline & $r \times 2 \mathrm{Lf}$ & TGCATGTTCTGGTTGCAACG & \multirow{2}{*}{$68^{\circ} \mathrm{C}$} & \multirow{2}{*}{44 secs } & \multirow{2}{*}{1457 bp } \\
\hline & gfp $r$ & AAACGCTCGACCAGGATGGGCA & & & \\
\hline & $r \times 2 \mathrm{Lf}$ & TGCATGTTCTGGTTGCAACG & \multirow{2}{*}{$67^{\circ} \mathrm{C}$} & \multirow{2}{*}{90 secs } & \multirow{2}{*}{$\begin{array}{l}1719 \mathrm{bp} \\
2547 \mathrm{bp}\end{array}$} \\
\hline & $r \times 2 \mathrm{Lr}$ & AGGGACCATACCTGACCCTC & & & \\
\hline & $a c t b \mathrm{Lf}$ & CAGCAACGACTTCGCACAAA & \multirow{2}{*}{$66^{\circ} \mathrm{C}$} & \multirow{2}{*}{24 secs } & \multirow{2}{*}{797 bp } \\
\hline & actb $\mathrm{Lr}$ & CAGGGGCAATTCTCAGCTCA & & & \\
\hline & $d n m t Y$ Lf & ACAGGTAAACCAGAAAAACTA & \multirow{2}{*}{$58^{\circ} \mathrm{C}$} & \multirow{2}{*}{11 secs } & $375 \mathrm{hn}$ \\
\hline & $d n m t Y L r$ & ААСТААТТСАТССССАТТСС & & & ת ס \\
\hline
\end{tabular}




\section{Genotyping}

298 Genotypes of samples used in our study were assessed based either on PCR band 299 size or using the T7 Endonuclease I Assay (New England Biolabs). However, in

300 order to save time, instead of purifying the PCR reactions, we directly transferred 10

$301 \mu \mathrm{l}$ of the unpurified PCR reaction into fresh PCR tubes with $7.5 \mu \mathrm{l}$ Milli-Q water and 2

$302 \mu \mathrm{l}$ NEB Buffer 2 (total $19.5 \mu \mathrm{l}$ ) and proceeded with heteroduplex formation (1:

303 Denature at $95{ }^{\circ} \mathrm{C}$ for 5 mins, 2: Stepwise cool down from $95^{\circ} \mathrm{C}$ to $85^{\circ} \mathrm{C}$ with -2

$304{ }^{\circ} \mathrm{C} / \mathrm{sec}$, and 3: Stepwise cool down from $85^{\circ} \mathrm{C}$ to $25^{\circ} \mathrm{C}$ with $-0.1^{\circ} \mathrm{C} / \mathrm{sec}$ ) and

305 subsequent digestion by adding $0.5 \mu \mathrm{l}$ of T7EI (NEB) and incubation for 30 min at 37

$306{ }^{\circ} \mathrm{C}$. After incubation, the digest was immediately run on a $1.5 \%$ Agarose in $1 \mathrm{XTAE}$ 307 gel at $90 \mathrm{~V}$.

\section{Acknowledgments}

310 We are grateful to F. Böttger for building a working copy of 'Thor's Hammer' for this

311 genotyping approach. Further we like to thank S. Lemke for Drosophila and

312 Chironomous flies and primers, M. Freichel for the mouse ear punch and primers, L.

313 Zilova for the mouse ES cells and J. Lohmann for the Arabidopsis seedlings and

314 primers. We further thank all members of the Wittbrodt lab for their critical,

315 constructive feedback on the procedure and the manuscript. $\mathrm{OH}$, member of the

316 Heidelberg International Biosciences Graduate School (HBIGS), was supported by a

317 fellowship of the DZHK. This work was supported by the coordinated program of the

318 DFG (FOR2509, project P10).

\section{Author contribution}


321 O.H., T.T. and J.W. designed the study, analysed the data and wrote the manuscript.

322 O.H. and T.T. performed injection experiments and acquired the data. O.H.

323 performed all genotyping and indel assay analyses.

324

\section{References}

326

327

328

329

330

331

332

333

334

335

336

337

338

339

340

341

342

343

344

345

346

347

348

349

350

351

352

353

354

355

356

357
Ali, N., Rampazzo, R. de C.P., Costa, A.D.T., and Krieger, M.A. (2017). Current Nucleic Acid Extraction Methods and Their Implications to Point-of-Care Diagnostics. BioMed Research International 2017, 9306564-13.

Baranski, T.J., Kraja, A.T., Fink, J.L., Feitosa, M., Lenzini, P.A., Borecki, I.B., Liu, C.T., Cupples, L.A., North, K.E., and Province, M.A. (2018). A high throughput, functional screen of human Body Mass Index GWAS loci using tissue-specific RNAi Drosophila melanogaster crosses. PLoS Genet 14, e1007222.

Bert, B., Chmielewska, J., Bergmann, S., Busch, M., Driever, W., Finger-Baier, K., Hößler, J., Köhler, A., Leich, N., Misgeld, T., et al. (2016). Considerations for a European animal welfare standard to evaluate adverse phenotypes in teleost fish. The EMBO Journal 35, 1151-1154.

Cong, L., Ran, F.A., Cox, D., Lin, S., Barretto, R., Habib, N., Hsu, P.D., Wu, X., Jiang, W., Marraffini, L.A., et al. (2013). Multiplex Genome Engineering Using CRISPR/Cas Systems. Science 339, 819-823.

Gutierrez-Triana, J.A., Tavhelidse, T., Thumberger, T., Thomas, I., Wittbrodt, B., Kellner, T., Anlas, K., Tsingos, E., and Wittbrodt, J. (2018). Efficient single-copy HDR by 5 ' modified long dsDNA donors. eLife 7, 142.

Jangam, S.R., Yamada, D.H., McFall, S.M., and Kelso, D.M. (2009). Rapid, point-ofcare extraction of human immunodeficiency virus type 1 proviral DNA from whole blood for detection by real-time PCR. J. Clin. Microbiol. 47, 2363-2368.

Kim, J., Mauk, M., Chen, D., Qiu, X., Kim, J., Gale, B., and Bau, H.H. (2010). A PCR reactor with an integrated alumina membrane for nucleic acid isolation. Analyst 135, 2408-2414.

Kirchmaier, S., Naruse, K., Wittbrodt, J., and Loosli, F. (2015). The genomic and genetic toolbox of the teleost medaka (Oryzias latipes). Genetics 199, 905-918.

Kithcart, A., and MacRae, C.A. (2017). Using Zebrafish for High-Throughput Screening of Novel Cardiovascular Drugs. JACC: Basic to Translational Science 2, $1-12$.

Köster, R., Stick, R., Loosli, F., and Wittbrodt, J. (1997). Medaka spalt acts as a target gene of hedgehog signaling. Development 124, 3147-3156.

Kweon, J., and Kim, Y. (2018). High-throughput genetic screens using CRISPR-Cas9 system. Arch. Pharm. Res. 41, 875-884. 
358 Lessman, C.A. (2011). The developing zebrafish (Danio rerio): A vertebrate model for 359 high-throughput screening of chemical libraries. Birth Defect Res C 93, 268-280.

360 Lischik, C.Q., Adelmann, L., and Wittbrodt, J. (2018). Enhanced in vivo-imaging in

361 fish by optimized anaesthesia, fluorescent protein selection and removal of

362 pigmentation. bioRxiv 428763.

363 Mali, P., Esvelt, K.M., and Church, G.M. (2013). Cas9 as a versatile tool for 364 engineering biology. Nat Meth 10, 957-963.

365 McFall, S.M., Wagner, R.L., Jangam, S.R., Yamada, D.H., Hardie, D., and Kelso, 366 D.M. (2015). A simple and rapid DNA extraction method from whole blood for highly 367 sensitive detection and quantitation of HIV-1 proviral DNA by real-time PCR. J. Virol. 368 Methods 214, 37-42.

369 Oxendine, S.L., Cowden, J., Hinton, D.E., and Padilla, S. (2006). Adapting the 370 medaka embryo assay to a high-throughput approach for developmental toxicity 371 testing. NeuroToxicology 27, 840-845.

372 Spivakov, M., Auer, T.O., Peravali, R., Dunham, I., Dolle, D., Fujiyama, A., Toyoda,

373 A., Aizu, T., Minakuchi, Y., Loosli, F., et al. (2014). Genomic and phenotypic

374 characterization of a wild medaka population: towards the establishment of an

375 isogenic population genetic resource in fish. G3 (Bethesda) 4, 433-445.

376 Spomer, W., Pfriem, A., Alshut, R., Just, S., and Pylatiuk, C. (2012). High-

377 Throughput Screening of Zebrafish Embryos Using Automated Heart Detection and

378 Imaging:. Journal of Laboratory Automation 17, 435-442.

379 Stemmer, M., Thumberger, T., del Sol Keyer, M., Wittbrodt, J., and Mateo, J.L.

380 (2015). CCTop: An Intuitive, Flexible and Reliable CRISPR/Cas9 Target Prediction

381 Tool. PLoS ONE 10, e0124633.

382 Thomsen, N., Ali, R.G., Ahmed, J.N., and Arkell, R.M. (2012). High resolution melt

383 analysis (HRMA); a viable alternative to agarose gel electrophoresis for mouse 384 genotyping. PLoS ONE 7, e45252.

385 Wittbrodt, J., Shima, A., and Schartl, M. (2002). MEDAKA - A MODEL ORGANISM

386 FROM THE FAR EAST. Nat. Rev. Genet. 3, 53-64.

387 Zischewski, J., Fischer, R., and Bortesi, L. (2017). Detection of on-target and off-

388 target mutations generated by CRISPR/Cas9 and other sequence-specific

389 nucleases. Biotechnol. Adv. 35, 95-104.

390 Zou, Y., Mason, M.G., Wang, Y., Wee, E., Turni, C., Blackall, P.J., Trau, M., and 391 Botella, J.R. (2017). Nucleic acid purification from plants, animals and microbes in 392 under 30 seconds. Plos Biol 15, e2003916. 


\section{Figure legends}

395 Figure 1. Filter-in-tips are highly sensitive for rapid transfer of nucleic acids for 396 targeted genome editing diagnostics

397 A) Assembly of filter-in-tip - Whatman filter paper / cellulose disc inserted into $200 \mu \mathrm{l}$ 398 yellow tip at the sample-proximal end. B) Genomic male sex determination by $d n m t Y$ 399 PCR of 3 male (M1-3) and 3 female (F1-3) fin clips. actb amplification as control, 400 purified and precipitated wt DNA as positive control (+), water as negative control (-).

401 Note: transfer of gDNA via filter-in-tips and specific amplification of loci was

402 successful in all cases. C) Schematic workflow of high-throughput 96-well plate based SLEDGE-Hammer protocol with filter-in-tips. Step 1 Lysis: (96-pin) mortar used to simultaneously grind individual embryos in fin-clip buffer. Step 2 Binding: pipet up lysate to let nucleic acids (red) bind to cellulose filter discs (soak for $\approx 10 \mathrm{sec}$ ) using a multichannel pipet equipped with filter-in-tips. Release lysate back in wells for storage. Step 3 Washing: wash filter discs containing nucleic acids (red) by pipetting nuclease free water in (wait $\approx 2-5 \mathrm{sec}$ ) and out. Step 4 Elution: pipet up pre-mixed PCR mixture (wait $\approx 5-10 \mathrm{sec}$ ) to release nucleic acids and pipet back for amplification. D) Schematic representation of CRISPR/Cas9 mediated NHEJ-based knock-out (medaka oca2, olOca2; zebrafish oca2, drOca2) and HDR-mediated

412 single-copy integration of gfp sequence in frame with medaka rx2 (cf. Gutierrez et al., 4132018 (Gutierrez-Triana et al., 2018)). Location of sgRNA target sites shown by 414 scissors. Primers used for analysis indicated; locus forward (Lf), locus reverse (Lr) of 415 the respective gene. $r \times 2$ locus shown after single-copy HDR-mediated integration of 416 gfp. E) Besides fully pigmented eyes in wt medaka and zebrafish, varying degrees of

417 pigment loss in body and RPE (blow-up) of representative crispants injected with 418 individual sgRNAs targeting oca2 exon 8. Retinae (dashed ellipses) of injected 419 embryos show GFP expressing cells after HDR/donor mediated integration of gfp into 
420 rx2 open reading frame. Note: unspecific autofluorescence of body pigment. 96-well

421 plate coordinates of specimens indicated (see Supplementary Fig. S1). F)

422 Genotyping of representative embryos in E. Locus PCR (L; primers Lf/Lr) of

423 respective genes and T7EI assay (T) validating indel formation in oca2 loci. In gfp-rx2

424 tagging, besides non-gfp-integrated locus band ( $L$; primers $L f / L r$ ), single gfp

425 integration evident by PCR (green asterisk). Integration validated by locus-gfp band

426 (G; primers Lf/gfp r).

427

428 Figure 2. General applicability of filter-in-tips to rapidly transfer genomic DNA

429 from various sources.

430 Filter-in-tips successfully transferred genomic DNA from lysates of Arabidopsis

431 thaliana $(A t)$, Chironomus riparius $(C r)$, Drosophila melanogaster $(D m)$, mouse (Mus

432 musculus, Mm) ear punches (EP) and mouse Embryonic Stem Cells (ESCs) as

433 evident by PCR amplification of candidate genes (tubulin; germ cell-less, gcl; twist;

434 trpm4). Water control (-).

435

436 Supplementary Figure 1. 96-well format high-throughput SLEDGE-Hammer

437 analysis

438 A) 96 well plate layout for high-throughput genotyping. CRISPR/Cas9 mediated

439 (green wells) knock-out of oca2 locus with three individual sgRNAs: olOca2 T1

440 (columns 1-3), olOca2 T2 (columns 4-6), drOca2 T1 (columns 7-9). HDR/donor

441 mediated integration of gfp in frame with rx2 locus (columns 10-12). Medium control

442 (blue wells) and uninjected wildtype specimens (red wells) included for control. B)

443 Successful rapid extraction/transfer of genomic DNA using filter-in-tips evident by

444 oca2 locus PCR amplification of injected and uninjected specimens. Larger random

445 indel formation can yield extra bands (black asterisks). C) T7El assay of locus 
446 amplification in B reveals specificity of genomic DNA transfer method by T7EI

447 digestion of heteroduplexes (cut bands) in oca2 crispants but not wildtype embryos.

448 D) rx2 locus PCR amplification of injected and uninjected specimens. Note: non-gfp-

449 integrated locus band (black arrowhead, $1719 \mathrm{bp}$ ) and single precise gfp integration

450 (green asterisks, 2547 bp) evident by band-size. Additional bands stem from NHEJ-

451 events. E) gfp-rx2 specific bands correlate with embryos expressing GFP in retinae.

452 All single-copy HDR-mediated gfp integration events in D could as well be verified by

453 band size (953 bp) here. In addition, some donors underwent NHEJ (red asterisk,

$454 \approx 1400 \mathrm{bp}$ ) or most probable concatenation events (yellow asterisks). 


\section{Hammouda et al. Figure2}

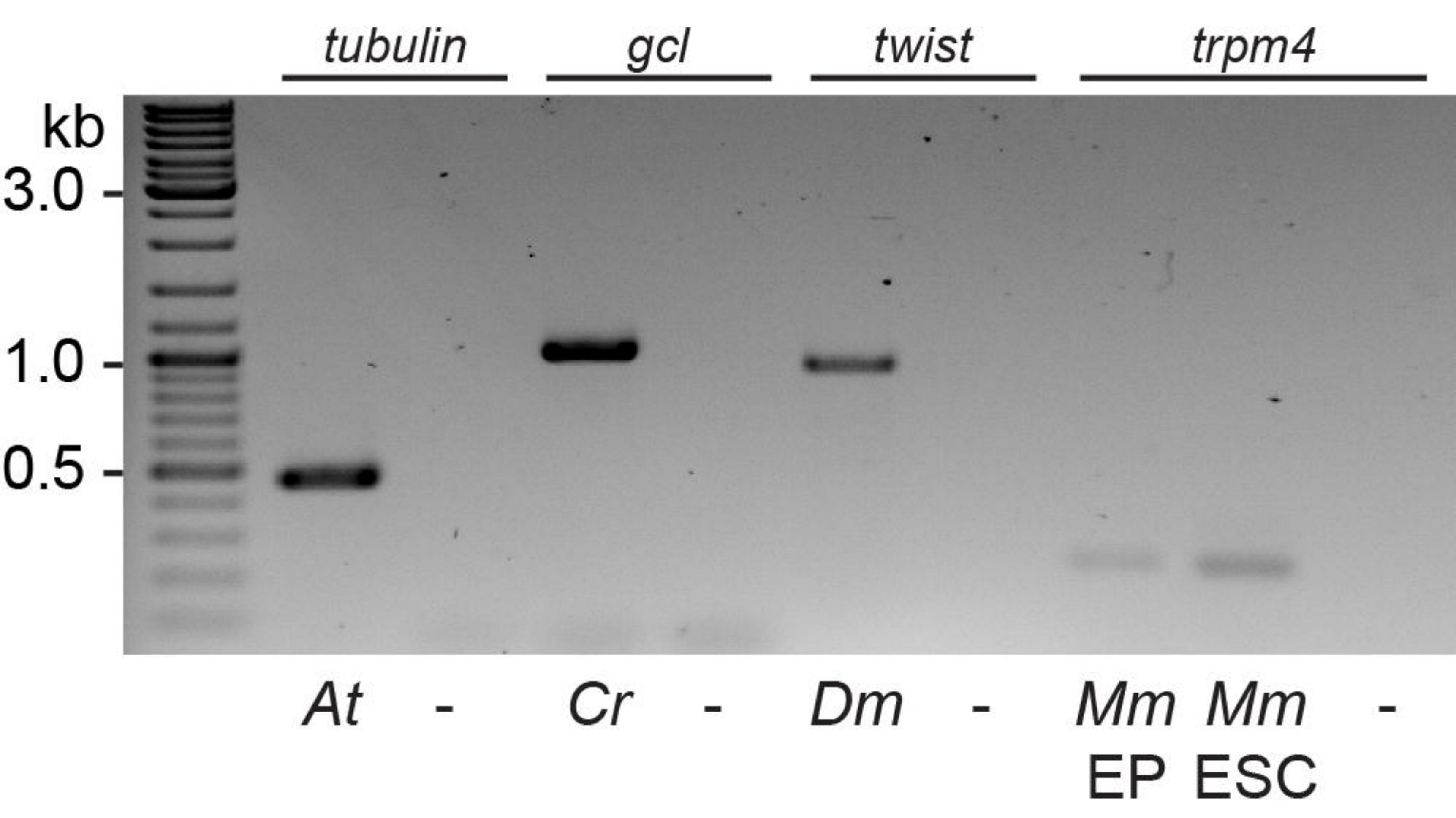

